\title{
Is video communication finally approaching Eldorado?
}

\author{
Stuart Gowland
}

What many people see as 20 years of false promises relating to the uses of video conferencing, and its underdelivery, has not led to a shortage of visionaries predicting great changes to the medical world.

The low take up has generally been the result of poor-quality pictures, frustrating audio delays, and its inconvenience, as physicians must travel distances to access the necessary equipment, which often doesn't work. Adequate-quality pictures were something that only television broadcasters could afford, but recently there have been changes. Modern computer networks allow 'network sharing', which is making good-quality video communication cheaper, although still not cheap enough for general use.

Let's say that the 'Eldorado' of video communication will allow you to 'teleport' yourself to another venue and easily collaborate with others, with pictures that reflect natural motion, faithfully displaying body language and gestures with good resolution. In order to get good pictures and minimal audio delay, the size of the information stream needs to be about $5 \mathrm{Mb} / \mathrm{s}$ (as with domestic television)-about 10 times that of conventional video conferencing and still, unfortunately, with a price to match.

Expanding on the positive side of the 'teleporting' concept, video collaboration might involve moving into a room, looking around, responding to a greeting, looking at or displaying $X$-rays, taking your place beside an operating table, looking down at the abdomen of a patient as laparoscopic ports are placed, and then looking down the laparoscope. It is now possible to be a remote, 'electronic' delegate or a remote, active participant in a workshop.

Technology to allow the control of multiple cameras and other devices has been available since the moon landing, but is only just being incorporated into video-terminal equipment. Luckily, this control requires minimal extra bandwidth compared with 'ever hungry' pictures. Robotic devices are a more recent
Natural-

quality video

communication

could be

achieved ...

[but] while the

technology

is here now, the electronic

roads are

still too

expensive...

$S$ Gowland is a

Urologist and the

Director of the Mobile

Surgical Services

Project, Christchurch, New Zealand.

\section{Competing interests}

The author declared he has no competing interests.

www.nature.com/clinicalpractice doi:10.1038/ncpuro0630 development and are already being used to perform surgery and other procedural tasks remotely. So far so good, but how do we connect using the networks available?

Networks, (i.e. the internet) create electronic pathways between towns, countries, and continents. These are WANs (wide area networks). Networks within buildings use preinstalled wiring and are called LANs (local area networks), and terminate in most offices at what are called Ethernet sockets. The LANs are operated and controlled in our institutions by IT departments that usually employ specialist network engineers. The networks are mostly used to link computers and sometimes phone systems, but can also be used to distribute video streams. Video streams run across WANs using a selection of what are called 'layers', which have multiplied and sometimes been superseded with time. The plethora of acronyms is confusing, but the fashionable layers in use are ATM (asynchronous transfer mode), MPLS (multi protocol label switching), and IP (internet protocol). The IP assigns telephone-type numbers to electronic devices, such as a video terminal, and is partly responsible for safely transferring video streams between terminals, simply by using a set of 'traffic' directions. Access can now be as simple as reaching for the telephone.

Video streams were originally designed for commercial television and had their own physical connections, which formed private networks. Sharing network space for 'small' users means putting an end to this, but security can still be maintained by forming a VPN (virtual private network). A VPN ensures that no connections can be made to or from the path as it accesses the internet.

Natural-quality video communication could be achieved, and be made accessible from your desk. Is it Eldorado? Probably not, as, while the technology is here now, the electronic roads are still too expensive for us. Pooling our requirements is the one way we could increase the volume of use, thereby forcing the price down. 